Simulation of Large Parallel Plasma Flows in the Tokamak SOL Driven by Cross-Field Transport Asymmetries

A. Yu. Pigarov, S. I. Krasheninnikov, B.

LaBombard, T. D. Rognlien

June 9, 2006

Journal of Nuclear Materials 
This document was prepared as an account of work sponsored by an agency of the United States Government. Neither the United States Government nor the University of California nor any of their employees, makes any warranty, express or implied, or assumes any legal liability or responsibility for the accuracy, completeness, or usefulness of any information, apparatus, product, or process disclosed, or represents that its use would not infringe privately owned rights. Reference herein to any specific commercial product, process, or service by trade name, trademark, manufacturer, or otherwise, does not necessarily constitute or imply its endorsement, recommendation, or favoring by the United States Government or the University of California. The views and opinions of authors expressed herein do not necessarily state or reflect those of the United States Government or the University of California, and shall not be used for advertising or product endorsement purposes. 


\title{
Simulation of large parallel plasma flows in the tokamak SOL driven by cross-field transport asymmetries**
}

\author{
A.Yu. Pigarov*a , S.I. Krasheninnikov ${ }^{\mathrm{a}}$, B. LaBombard ${ }^{\mathrm{b}}$, T.D. Rognlien ${ }^{\mathrm{c}}$ \\ ${ }^{a}$ University of California at San Diego, La Jolla CA, USA \\ ${ }^{b}$ Massachusetts Institute of Technology, Cambridge, Massachusetts, USA \\ ${ }^{b}$ Lawrence Livermore National Laboratory, Livermore CA, USA
}

\begin{abstract}
Large-Mach-number parallel plasma flows in the single-null SOL of different tokamaks are simulated with multi-fluid transport code UEDGE. The key role of poloidal asymmetry of cross-field plasma transport as the driving mechanism for such flows is discussed. The impact of ballooning-like diffusive and convective transport and plasma flows on divertor detachment, material migration, impurity flows, and erosion/deposition profiles is studied. The results on well-balanced double null plasma modeling that are indicative of strong asymmetry of cross-field transport are presented.
\end{abstract}

PACS: 52.55.Fa, 52.40.Hf, 52.25.Vy, 52.30Ex, 52.30.-q

JNM keywords: T0100, R0900, I0100

PSI-17 keywords: Cross-field transport, Plasma flow, Edge plasma, Impurity transport, UEDGE

* Corresponding author address: UCSD Center for Energy Research, EBU-II, 9500 Gilman

Dr., La Jolla, CA 9209-0417, USA

Corresponding author E-mail: apigarov@uscd.edu

**Work by one of the authors (TDR) was performed under the auspices of the U.S. Dept. of Energy by the University of California Lawrence Livermore National Laboratory $\square$ under contract No. W-7405-Eng-48. 


\section{Introduction}

Recent experiments on several tokamaks and supporting theoretical studies have showed the strong cross-field plasma convection (due to intermittent edge turbulence) as well as the existence of large parallel plasma flows (LPPFs) in the SOL of single null (SN) and unbalanced double null (UDN) magnetic configurations. Detailed experimental characterization of SN and UDN discharges in Alcator C-Mod indicates [1] that LPPFs originate in the SOL near the low field side (LFS) mid-plane and accelerate to near sonic speed at the high field side (HFS) mid-plane toward the HFS divertor. These C-Mod observations and some related experiments on other tokamaks have revealed an important edge-physics phenomenon: the dominant driving mechanism of LPPFs appears to be a strong ballooning-like asymmetry in the anomalous cross-field (ACF) plasma transport. Moreover, substantially different radial profiles of plasma parameters measured at the LFS and HFS midplanes (MP) in the well-balanced DN discharges on C-Mod can also be explained by the strong LFS/HFS asymmetry in ACF transport.

We present results of multi-fluid modeling with UEDGE code of large parallel plasma flows in the SOL that demonstrate the outstanding role of ballooning-like asymmetry in crossfield plasma transport as the driving mechanisms of such flows. The impact of LPPFs on divertor detachment, material migration, impurity flows, and erosion/deposition profiles is studied.

\section{Anomalous cross-field transport model in UEDGE}

The UEDGE code is used for extensive simulations of edge plasma and neutral gas transport (including multi-species, multi-ion, fluid equations along magnetic field lines, realistic non-orthogonal 2D mesh based on tokamak equilibrium). We employ diffusive-and- 
convective model [2] for ACF plasma transport. Intermittent (e.g. due to blobs and holes) transport effect is modeled by means of anomalous convective velocity $\mathrm{V}_{\text {conv. Ion charge }}$ states may have different sign and amplitude of $\mathrm{V}_{\text {conv }}$ [2]. The model introduces asymmetric (e.g. ballooning-like) 2D profiles for all transport coefficients, which are adjusted to match a representative set of experimental data. Being a smooth function along the magnetic surface, the profile of $\mathrm{V}_{\text {conv }}$ is characterized by asymmetry factor $\eta_{\mathrm{as}}=\mathrm{V}_{\text {conv }}(\mathrm{IMP}) / \mathrm{V}_{\text {conv }}(\mathrm{OMP})$, where IMP and OMP stand for the inner and outer mid-planes. Profiles of plasma diffusivities may have their own asymmetry factors. No classical drifts are used here and a discussion on the effect of drifts on LPPFs will be presented in elsewhere.

\section{Cross-field transport asymmetry as the LPPF driving mechanism}

In our model, the cross-field transport, which is mainly due to plasma convection by blobs in the SOL, is strongly peaked around the LFS mid-plane with magnitude of $\mathrm{V}_{\text {conv }}$ increasing in the radial direction. At the wall, the peak $\mathrm{V}_{\text {conv }}$ typically is a few hundred $\mathrm{m} / \mathrm{s}$. The fast and spatially localized transport pushes plasma into the far SOL, so that, the total (gas+plasma) pressure profile along magnetic field lines may have pronounced maximum $(1.5-2 \mathrm{X})$ at the OMP, thus, causing the parallel plasma flows into the inner and outer divertors. This flow driving mechanism is demonstrated on Fig.1 by displaying some results of UEDGE modeling of the C-Mod discharge having been considered in [3]. Inspecting

profiles of plasma parameters, total pressure, velocity $\mathrm{V}_{\|}$, Mach number $\mathrm{M}_{\|}$, and flux $\Phi_{\|}$of plasma in parallel direction and comparing the profiles in the far SOL to the profiles adjacent to separatrix, one sees that (i) plasma flow at the magnetic surface closer to separatrix results in the plasma pressure equilibration due to high recycling on the divertor plates followed by the flow stagnation (notice, the OMP pressure maximum is small, since $n_{e}$ decrease is 
compensated by increase in $\mathrm{T}_{\mathrm{e}}+\mathrm{T}_{\mathrm{i}}$ ), whereas (ii) plasma flow at the magnetic surface in the far SOL accelerates to about sonic speed by parallel pressure gradient due to relatively weak plasma collisionality, low viscosity, and easier leakage of neutral (or plasma) particles back to core from inner divertor (notice, at the OMP both $\mathrm{n}_{\mathrm{e}}$ and $\mathrm{T}_{\mathrm{e}}$ are peaked maintaining the pressure maximum).

As simulated with UEDGE, the LFS-to-HFS plasma flow exists in SN in a wide range of $\eta_{\text {as }}<0.8$. However, only low values $\eta_{\text {as }} \approx 0.1$ (leading to large LFS/HFS flux asymmetry $\Phi_{\mathrm{LFS}} /\left|\Phi_{\mathrm{HFS}}\right|>70$ ) are consistent with experimental data as shown on Fig.2 (left) for this C-Mod discharge. Note, that we use the modulus of particle flux coupled to the HFS area of separatrix, since at low $\eta_{\text {as }}$ net particle flux $\Phi_{\mathrm{HFS}}<0$ is into the core due to plasma backflow from the inner divertor near the separatrix. The variation of LPPFs characteristics with $\eta_{\text {as }}$ is shown on Fig.2 (right). As seen, the LPPF carries $\approx 20 \%$ of plasma particles and energy entering the SOL through the separatrix. At large flux asymmetries $\Phi_{\mathrm{LFS}} /\left|\Phi_{\mathrm{HFS}}\right|$ (i.e. low $\eta_{\mathrm{as}}$ ) the particle flux is relatively small, so that for the given power input, the inner divertor is attached as it is in this discharge. In this case, flow velocity is the highest and maximal $\mathrm{M}_{\|}$can reach unity. With increasing in $\eta_{\text {as }}$, primarily results in a strong increase of particle flux into the inner divertor, the IMP radial profile of plasma density broadens and the plasma collisionality and divertor opacity to neutrals increase in the entire SOL, so that, the plasma flow tends to stagnate also in the far SOL region. In the case when ACF transport is more or less poloidally symmetric, the inner divertor leg is detached.

\section{Role of LPPF in impurity migration and deposition}

Impurity sources and parallel flows of impurity ions for LPPF conditions are simulated with UEDGE under the assumption of high LFS/HFS cross-field transport asymmetry. The 
impurity cross-field transport model assigns different anomalous convective velocities to charge states of plasma ions (parameter $\eta_{\mathrm{CCC}}=-1,[2]$ ) in order to match various experimental data. For L-mode discharges on NSTX, C-Mod and DIII-D tokamaks, our simulations have showed that high charge states $(Z>2)$ of impurity ions are highly entrained by the background deuterium plasma flows from near the outer mid-plane into the divertors, as shown in Fig.3 (right). The flow pattern of the lowest charge states, $\mathrm{C}^{+1}$ and $\mathrm{C}^{+2}$, is more complicated since they are also affected by the impurity sources and strong cross-field convective transport. In the far-SOL region when LPPFs are the strongest, Fig. 3 (left) shows that the impurity ions tend to have the same flow velocity as the deuterium ions, so that impurities are highly supersonic when the deuterium plasma flow is near-sonic.

For the typical LSN shot on C-Mod with LPPF, the diagram of impurity fluxes inferred from UEDGE simulations is shown in Fig. 4 (middle). As seen, the outer chamber wall exhibits large net erosion $(21.4-5.12=16.3 \mathrm{~A})$. This strong erosion is due to sputtering by intermittent convective cross-field plasma flux. In material migration analysis, the outer wall is the dominant donor of impurities, whereas the outer and inner divertor plates are the net sinks for impurities. The net particle deposition on the outer and inner plates are 11 and $5 \mathrm{~A}$, respectively, and their sum, $16 \mathrm{~A}$, is roughly equal to the wall net erosion. Large parallel plasma flow carries $5 \mathrm{~A}$ into the inner divertor plate, that is, $\sim 20 \%$ of all impurity ions sputtered from the outer wall. The material sputtered from outer wall (11A) consists of 25\% of the outer plate erosion rate (42A). Impurities from the outer wall are shared between inner and outer divertors roughly in the same ratio as the cross-field plasma flux does when passing through separatrix.

The UEDGE modeling of this shot also shows that impurities sputtered from outer chamber wall are transported by LPPF into the inner divertor mainly in the far SOL region. The calculated profiles of impurity erosion and deposition at the inner plate are shown in 
Fig.4 (right). As seen, in the far SOL, $\rho>0.5$, the erosion rate decreases with $\rho$ since the plasma temperature and flux decrease there, whereas the deposition rate is realatively constant. Erosion/deposition rates are largely peaked near the strike points, whereas the net deposition is small compared to the deposition in the far SOL. As modeled, the largest net deposition rates are attained in the far SOL region of both inner and ourter divertor plates. The net deposition profile predicted by UEDGE to be peaked in the far SOL region is in agreement with some tokamak experiments, for example, Be deposition on $\mathrm{C}$ in the inner divertor in JET [4] and $\mathrm{W}$ on $\mathrm{C}$ in ASDEX. The impurity deposition into this region can result is enhanced growth of dust particles [5] and, since dust particles transfer their mass predominantly from inner to outer divertor, dust may help form loop for material migration.

\section{Cross-field transport asymmetry in DN configuration}

Experiments with an unbalanced double null magnetic configuration on C-Mod show (as measured by reciprocating probes at the LFS and HFS mid-planes) that plasma density profiles have a much shorter cross-field decay length in the region outside the secondary separatrix on the HFS compared to the same region on the LFS [6]. In well-balanced DN discharges, the density e-folding length measured at the HFS mid-plane is substantially smaller than that in the lower single null magnetic configuration (Fig. 5). These data independently indicate a strong ballooning-like asymmetry where the cross-field plasma flux is much larger on the LFS.

UEDGE modeling of a well-balanced double-null shot obtained in C-mod is used to infer an asymmetry factors for the anomalous cross-field plasma (diffusive and convective) transport from matching experimental profile data. Firstly, for fixed $D_{\perp}$ and $\chi_{\perp}$, we vary the asymmetry of convective velocity in two ways: (i) scan values of $\mathrm{V}_{\text {conv }}$ (IMP) at inner mid- 
plane while $\mathrm{V}_{\text {conv }}(\mathrm{OMP})$ at outer mid-plane is fixed, and (ii) vary $\mathrm{V}_{\text {conv }}(\mathrm{OMP})$ and asymmetry factors $\eta_{\mathrm{as}}=\mathrm{V}_{\text {conv }}(\mathrm{IMP}) / \mathrm{V}_{\text {conv }}(\mathrm{OMP})$ and select runs with the same total plasma flux through the separatrix where the flux is approximately given by ionization source from $D_{\alpha}$ measurements and by pressure measurements at outer mid-plane. In both cases we find that small asymmetries, i.e. $\eta_{\mathrm{as}}>0.1$, substantially overestimate the plasma density in the far SOL at HFS as well as the $\mathrm{J}_{\text {sat }}$ at inner plate. This result is exactly what one should expect from the very nature of blobby transport since in DN the LFS and HFS parts of SOL are completely disconnected. Secondly, we vary the peak value and asymmetry in profiles of all transport coefficients $\left(\mathrm{V}_{\text {conv }}, \mathrm{D}_{\perp}\right.$, and $\left.\chi_{\perp}\right)$ and obtain the best fit of UEDGE to experimental data. The best-fit results are shown in Fig.5 for the LFS, HFS, and divertor probes data. In this case, convective transport at the HFS is small, $\mathrm{V}_{\text {conv }}(\mathrm{IMP}) / \mathrm{V}_{\text {conv }}(\mathrm{OMP})=0.05$ (or even no blobby transport), and the asymmetry in plasma diffusivities $\eta_{\text {as }}(D, \chi)=0.2$ is also small. Notice that experimental profiles are reproduced by UEDGE reasonably well in a wide range $0.1<\eta_{\text {as }}(D, \chi)<0.4$, and the accuracy of experimental data does not allow a better resolution of transport asymmetry. The smaller asymmetry factors $\eta_{\mathrm{as}}(\mathrm{D}, \chi)<0.1$ result in the inner leg detachment since small power is transported into the HFS SOL, whereas the values larger than 0.4 cause, e.g., significant overestimation of $\mathrm{J}_{\text {sat }}$ at HFS. Finally, our previous study of LSN discharge on C-Mod found that the ballooning-like asymmetries were necessary assigned in LSN to both $\mathrm{V}_{\text {conv }}$ and diffusivities. The asymmetry factors deduced from matching all 5 probe systems on C-Mod in this shot with UEDGE were in the range $\eta_{\text {as }}\left(V_{\text {conv }}\right)=\eta_{\text {as }}(D, \chi)=$ 0.05-0.15 which is consistent with our results for the present DN case.

\section{References}

[1] B. LaBombard et al, Nucl. Fusion 44 (2004) 1047 
[2] A.Yu. Pigarov et al, J. Nucl. Materials 337-339 (2005) 371

[3] A.Yu. Pigarov et al, Contrib. Plasma Phys. 46 (2006)

[4] M. Rubel et al, J. Nucl. Materials 313-316 (2003) 321

[5] A.Yu Pigarov et al, "Dust particle transport in tokamak devices", J. Nucl. Materials, this issue.

[6] C.J. Boswell et al, Plasma Phys. Control. Fusion 46 (2004) 1247

\section{Figure captions:}

Fig.1: Plasma parameter profiles along magnetic field lines (MFLs) in the SOL (0 and 1 ordinate values correspond to the inner and outer divertor plates) calculated with UEDGE, $\eta_{\text {as }}=0.1$, for a LSN shot on C-Mod. Broken curves show the profiles for a MFL in the middle of SOL $(\rho=0.5 \mathrm{~cm})$, whereas solid curves represent profiles in the far SOL $(\rho=1.3 \mathrm{~cm})$. Vertical lines correspond to locations of (from left to right) entrance to inner divertor, inner MP, plasma top, outer MP, and entrance to outer divertor. Negative values of $\mathrm{V}_{\|}, \mathrm{M}_{\|}$, and $\Phi_{\|}$ correspond to the flow toward the inner plate.

Fig.2: The maximal $T_{e}$ at the inner plate, $M_{\|}$, and the ratio $\Phi_{\mathrm{LFS}} /\left|\Phi_{\mathrm{HFS}}\right|$ are displayed versus asymmetry factor $\eta_{\mathrm{as}}$ of cross-field transport on the left panels. The UEDGE calculations were done for typical C-Mod L-mode shot. Experimental data are scattered within two horizontal lines. In the right panels, the calculated ratios $\left(\Phi_{\mathrm{TOP}} / \Phi_{\mathrm{SEP}}\right.$ and $\mathrm{q}_{\mathrm{TOP}} / \mathrm{q}_{\mathrm{SEP}}$, where $\Phi_{\mathrm{TOP}}, \mathrm{q}_{\mathrm{TOP}}$ and $\Phi_{\mathrm{SEP}}, \mathrm{q}_{\mathrm{SEP}}$ are the total particle and energy fluxes carried by the LPPF and transported through separatrix), number of particles in the inner divertor, and averaged SOL plasma density are shown versus $\Phi_{\mathrm{LFS}} /\left|\Phi_{\mathrm{HFS}}\right|$, the real LFS/HFS flux asymmetry. 
Fig.3: Radial profiles of flow velocities are shown for background plasma and for different impurity ion charge states on the left panel. These profiles are related to SOL at the inner midplane, whereas the coordinate $\rho$ is mapping the magnetic flux surfaces to their distance from separatrix at the LFS mid-plane. The variations of these velocities with distance along the magnetic field line from inner to outer divertor plates in the far SOL $(\rho=0.8 \mathrm{~cm})$.

Fig.4: The impurity flow diagram calculated with UEDGE (middle panel). The letters stand for characteristic points in the computational mesh (left). Radial profiles of erosion (negative curve), deposition (positive), and net deposition rates at the inner divertor plate are displayed on the right panel as functions of $\rho$, the distance from separatrix to magnetic flux surface at the outer mid-plane, $\rho>0$ correspond to the SOL.

Fig.5: The results of UEDGE simulations are compared with experimental profiles of plasma density $n_{e}$, ion saturation current $J_{\text {sat }}$, and temperature $T_{e}$ at the LFS and HFS mid-planes and at the HFS plate as measured by reciprocating and Langmuir probes in C-Mod for wellbalanced DN. 

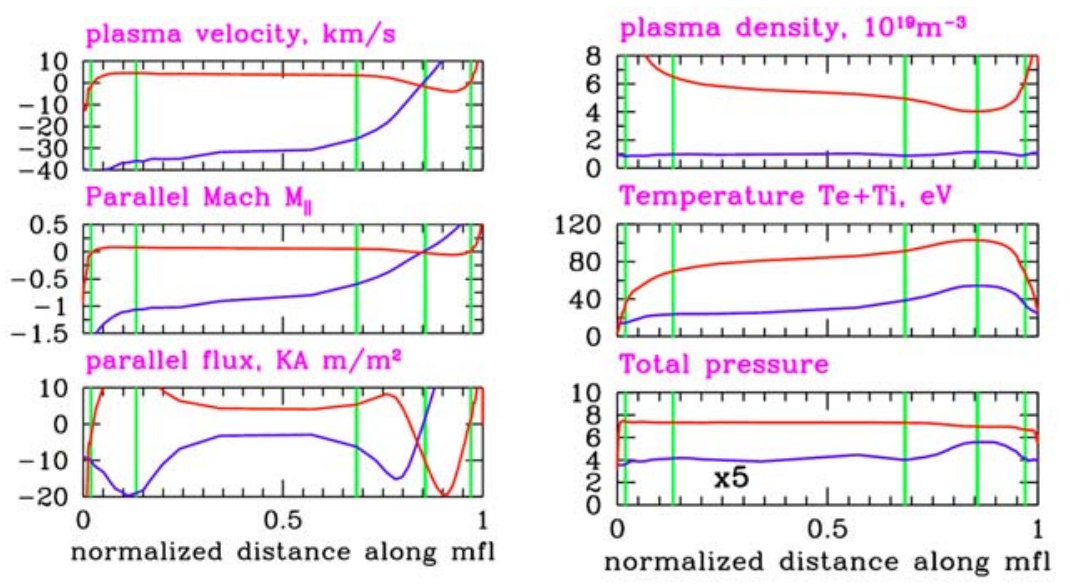

Fig. 1
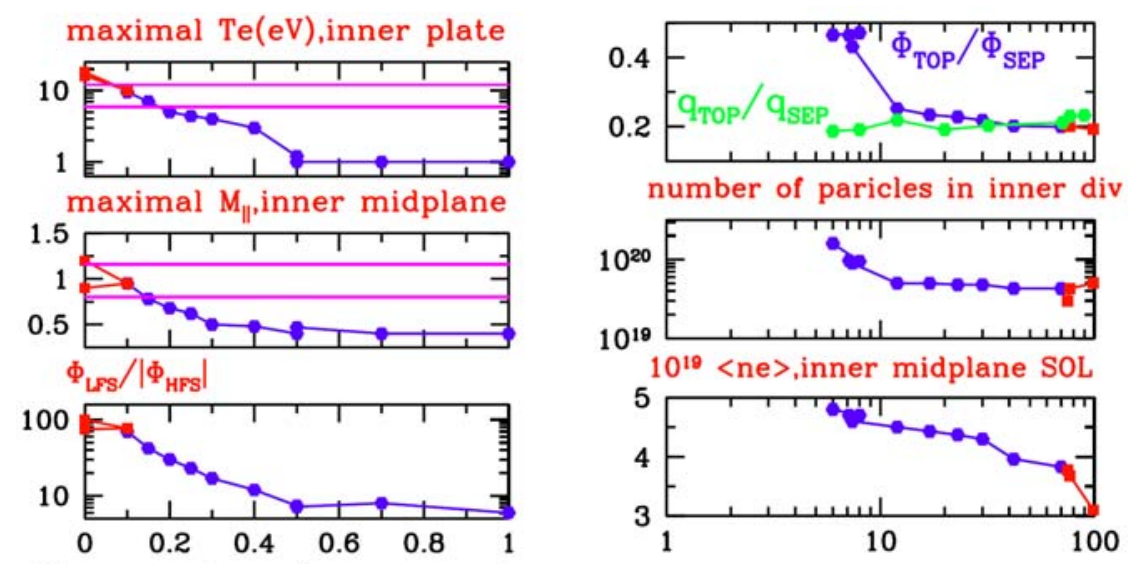

Transport coefs. asymmetry

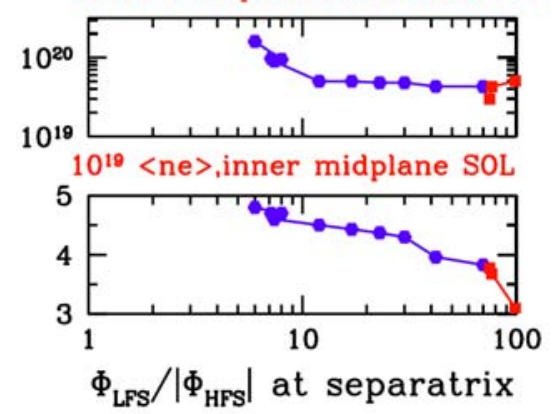

Fig. 2 

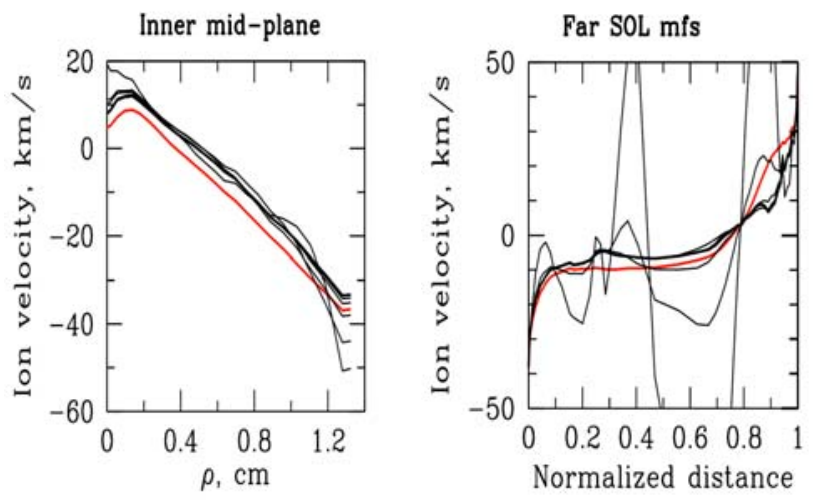

Fig. 3
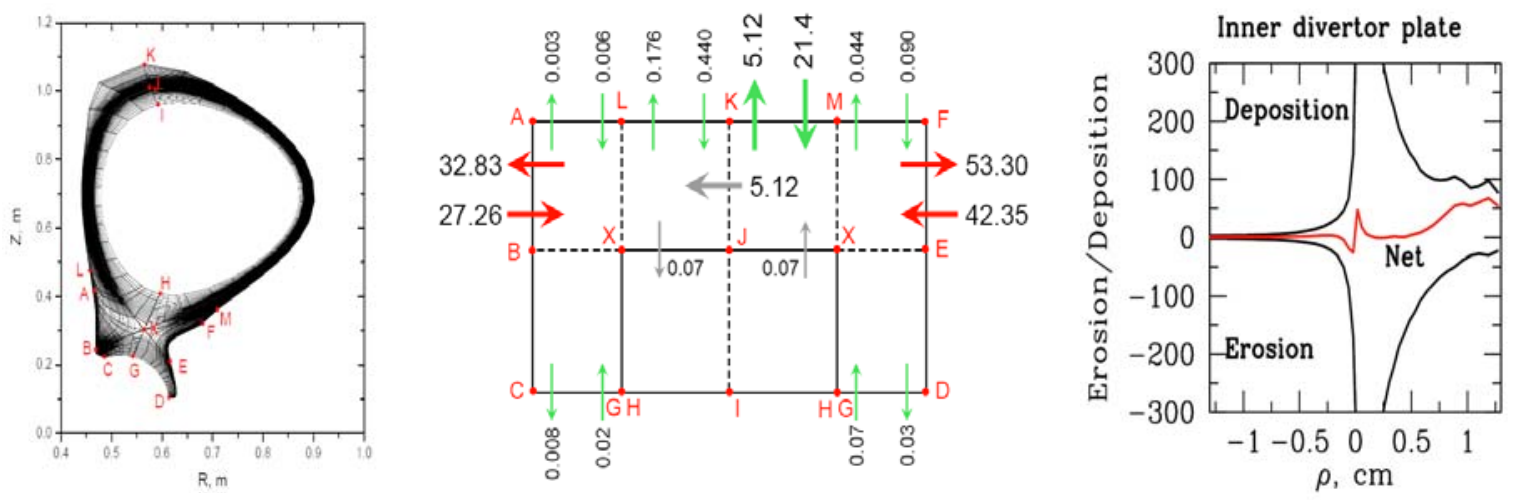

Fig.4

Te, eV, OUTER mid-plane
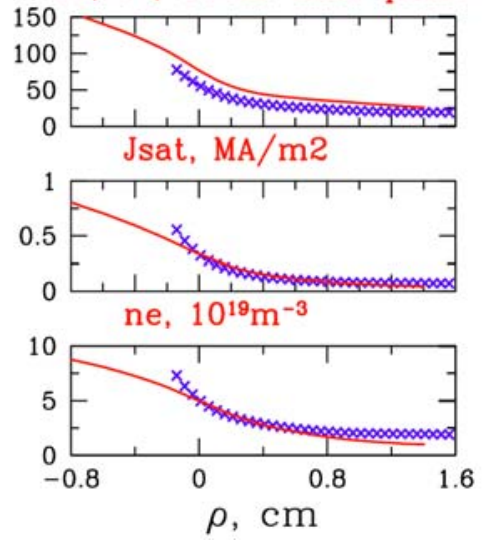

Te, eV, INNER mid-plane
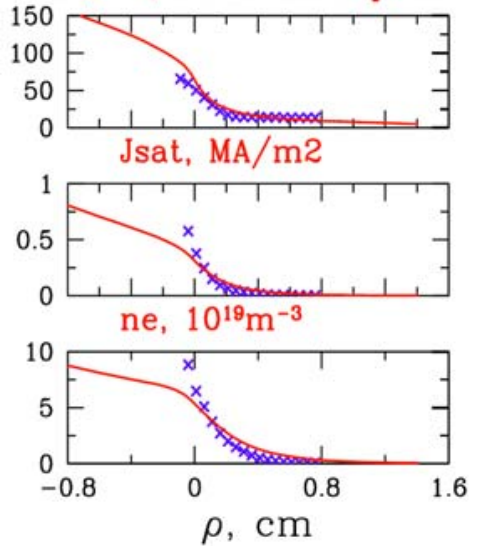

$\mathrm{Te}, \mathrm{eV}$, INNER PLATE
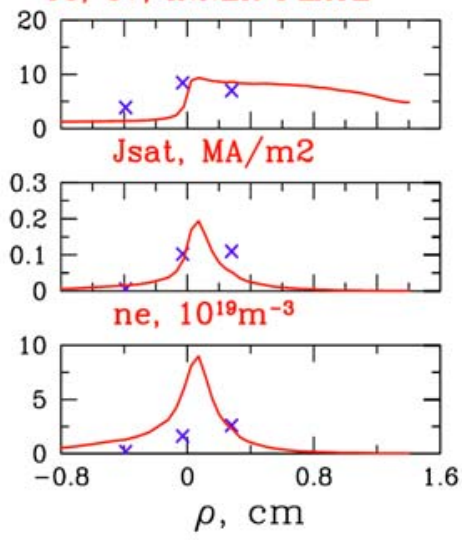

Fig. 5 